\title{
Synchronization between two discrete chaotic systems for secure communications
}

\begin{abstract}
In this article, we have investigated synchronization phenomenon between two discrete chaotic systems. A general scheme for synchronization between two discrete maps with adaptive coupling has been studied analytically. The scheme can be successfully implemented for generalized synchronization between two chaotic maps. Conditional Lyapunov exponents (CLE) and Transverse Lyapunov exponents (TLE) can quantifies the robustness of synchronization. A secure communication scheme based on synchronization between two Logistic maps is also demonstrated. Numerical results show the effectiveness of our proposed scheme.
\end{abstract}

Keyword: Synchronization; Secure communication; Chaotic systems 